\section{Magnetic Confinement Fusion}

\author{
David Campbell \\ The NET Team, Max-Planck-Institut für Plasmaphysik, Garching, Germany
}

\begin{abstract}
Left A schematic of the Wendelstein In the course of the last 50 years research on magnetically confined plasmas has brought magnetic confinement fusion to the threshold of net power pro-

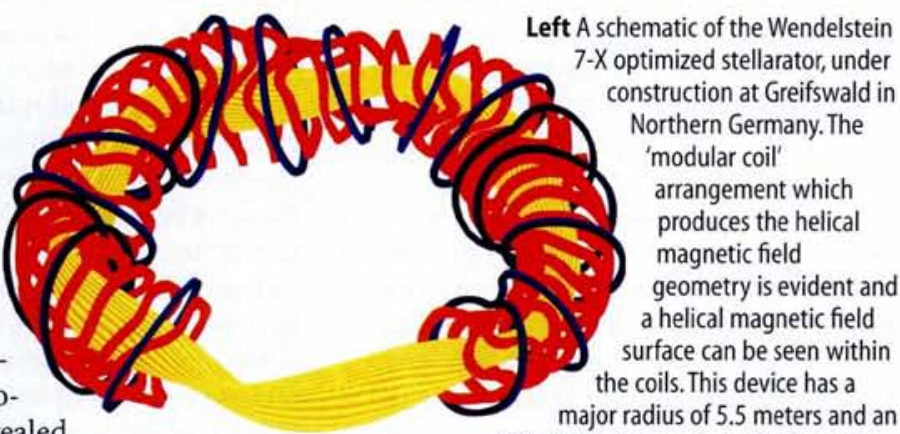
duction and has revealed
\end{abstract} much of the physics underlying the astonishingly complex behaviour of hot plasmas immersed in a magnetic field.

The simplest approach to magnetic confinement uses the longitudinal field inside a long straight solenoid. Unfortunately, the rapid transport of particles along the field lines leads to unsustainable losses from the ends of the configuration. If the solenoid is bent into a doughnut, or torus, the problem of end losses immediately disappears. However, a toroidal magnetic field is curved and its magnitude falls off with increasing radius from the centre of the torus. Both these properties cause charged particles to drift up or down across the field lines. Since ions and electrons drift in opposite directions a large electric field develops further enhancing the loss of plasma across field lines. As shown in figure 1a, which defines key parameters of toroidal geometry, a poloidal magnetic field, acting at right angles to the toroidal field, can be added. The resultant field consists of a set of nested helical field lines, and as the particles follow these helical trajectories around the torus, the drifts are cancelled producing a well confined magnetoplasma.

The focus of contemporary fusion research is the deuterium-tritium reaction

${ }_{1} \mathrm{D}^{2}+{ }_{1} \mathrm{~T}^{3} \rightarrow{ }_{2} \mathrm{He}^{4}(3.5 \mathrm{MeV})+{ }_{0} \mathrm{n}^{1}(14.1 \mathrm{MeV})$

which is the fusion reaction with the largest cross-section at the temperatures which are likely to be achieved in laboratory experiments (several $10^{8} \mathrm{~K}$ ). Eighty per cent of the reaction energy appears as neutrons are produced, or in deuterium, for which the neutron production rate is almost two orders of magnitude lower than in a deuterium-tritium mixture.

To achieve the conditions necessary for 'ignition', where the $\alpha$-particle power produced by fusion reactions exactly balances the heat loss due to transport processes, the plasma must be heated to a temperature of approximately $10^{8} \mathrm{~K}$ (nearly a factor of 10 greater than the temperature at the centre of the sun) at a particle density in the region of $10^{20}$ ions per cubic metre (about $10^{-6}$ the density of air), while maintaining an energy replacement time of about 5 seconds. The energy replacement time is a measure of the confinement quality of the plasma system and it is defined by the ratio of the thermal energy stored in the plasma to the power lost by conduction and convection. This should not be confused with plasma lifetime, which is usually much longer. How the required plasma conditions are attained, the prospects for maintaining them in steady-state, and the processes that affect confinement quality, plasma stability, and plasma-wall interactions are outlined in the following sections. power plant and provide most of the energy for steam generation. The $\alpha$-particle would be trapped in the plasma where its energy would heat the plasma and maintain the conditions required for fusion reactions to occur. Since tritium is a radioactive gas and since a high flux of 14.1 MeV neutrons would induce significant radioactivity in the structure surrounding the plasma, current experiments on magnetically confined plasmas are usually carried out in hydrogen, so that no

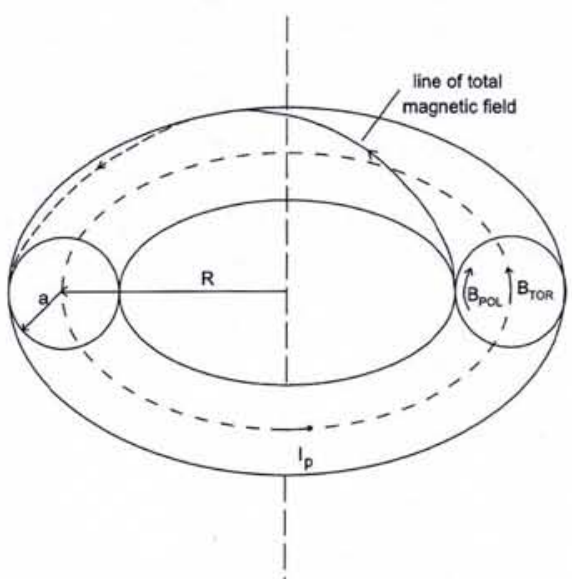

Fig 1a Schematic of a toroidal magnetic confinement system illustrating the principal parameters and the magnetic geometry. The distance from the central axis of symmetry to the centre of the toroidal ring is the major radius, $R$, and the distance from the centre of the toroidal ring to its edge is the minor radius, $a$. The ratio $R / a$ is known as the 'aspect ratio'. The toroidal field, $B_{\text {tor }}$ is directed around the long circumference of the torus

\section{Toroidal magnetic confinement}

Three principal toroidal confinement systems have emerged as potential routes to a fusion power plant. In the tokamak, figure $\mathrm{ib}$, a strong toroidal field of several Tesla is produced by a set of discrete coils. Transformer coupling between a vertical solenoid which is wound inside the central hole of the torus and the toroidal ring of

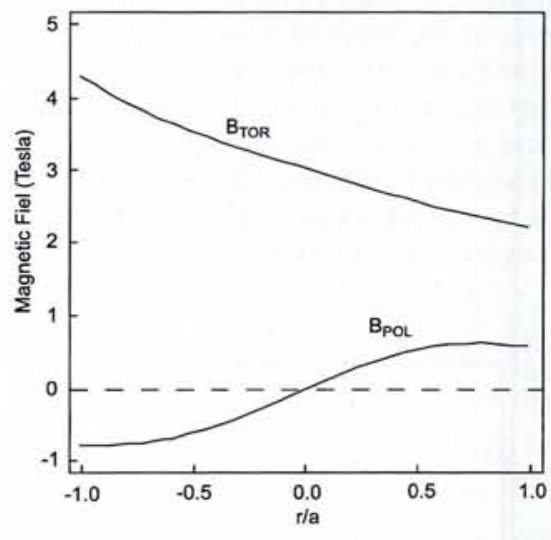

1b Spatial variation of the toroidal and poloidal fields across the poloidal cross-section of the torus in a tokamak. Note the difference in magnitude of the two while the poloidal field, $\mathrm{B}_{\text {por }}$ is directed around the short circumference. The field line corresponding to the total field then forms a helix around the torus, and the magnetic configuration consists of a set of nested helices with different pitch angles see colour illustration above 


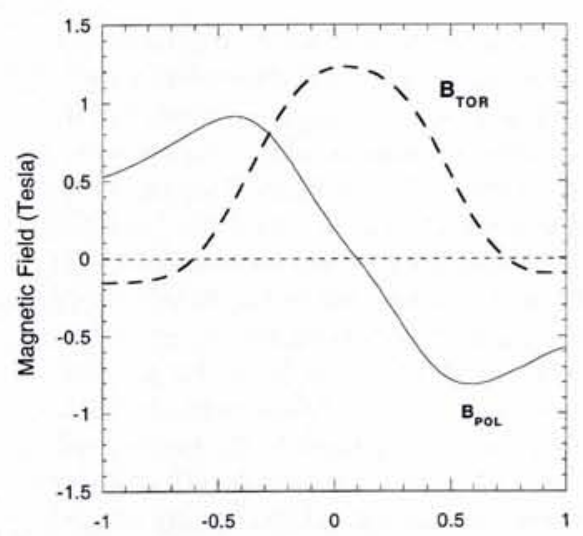

Fig 1c Spatial variation of the toroidal and poloidal fields in a reversed field pinch. Note that the two are of similar magnitude and that the toroidal field changes sign close to the plasma edge (courtesy D.E. Baker and W.E. Quinn, Fusion, edited by E Teller, Academic Press, New York, 1981, 437-475)

plasma induces a toroidal current. This produces the poloidal component of the magnetic field and also raises the plasma temperature, to perhaps several $\mathrm{keV}$, by Joule (or ohmic) heating $(1 \mathrm{keV}=$ $1.16 \times 10^{7} \mathrm{~K}$ is the conventional unit of temperature in fusion research). Additional poloidal field components can then be applied by external coils to shape the plasma and control its position.

The reversed field pinch (RFP) is a closely related configuration, since the plasma formation and ohmic heating are essentially identical to the tokamak. However, in the tokamak the average poloidal field is limited by stability requirements to approximately an order of magnitude smaller than the toroidal field, whereas the two are of similar magnitude in the RFP, both being typically less than 1 Tesla (figure 1c). Moreover, to provide stability the toroidal field must reverse sign at the edge of an RFP (hence the name) and this occurs as a result of poloidal currents which develop inside the plasma due to a magnetic relaxation process. This is referred to as the RFP dynamo and it leads to a minimum energy state for the configuration.

The helical field in toroidal configurations can be described by the 'safety factor' $q$ (or by the rotational transform $l=$ $2 \pi / q$ ). This is most simply thought of as a 'magnetic winding number' which gives the number of times a helical field line must circle the torus toroidally before it returns to the same position in the poloidal plane. Since the poloidal and toroidal fields are of the same order in the RFP, $q<1$ everywhere and falls towards the plasma edge, whereas in the tokamak $q$ is of order unity at the plasma centre and rises to 3-5 near the plasma edge. The absolute value of $q$ and the way in which it varies across the plasma cross-section have important consequences for the growth of instabilities in the plasma ring.

The third class of toroidal confinement devices is the stellarator (figure 1d) which differs in an essential way from tokamaks and RFPs in that the helical fields are created entirely by coils external to the plasma, with no net toroidal current flowing within the plasma (see Alejaldre, chapter 5.1). In a stellarator, the magnitudes of the toroidal and poloidal fields are similar to those of a tokamak and thus the range of $q$ values in the plasma is of the same order in the two devices. However, in the stellarator $q$ has a weak radial variation and falls towards the plasma edge.

In terms of power plant constraints the absence of a net toroidal current is a particularly attractive feature of stellarators. However, the stellarator plasma forms a toroidally periodic, three-dimensional structure (as shown in the colour illustration on the previous page) whereas tokamak and RFP plasmas consist of a toroidal ring of circular or elliptical poloidal crosssection within which helical magnetic field lines are embedded, as in figure 1a. This additional complexity degrades the plasma confinement properties of a stellarator, but the identification, in the 1980s, of a class of helical geometries which approximate the intrinsic confinement properties of tokamaks revitalized stellarator research (see Nührenberg, chapter 3.4). An additional and significant advantage of these 'advanced stellarators' is that the magnetic configuration can be established by a toroidally distributed set of 'modular' coils (see previous page).

At present the development of plasma parameters required for power production is most advanced in the tokamak. This is due, in part, to the increased focus on the

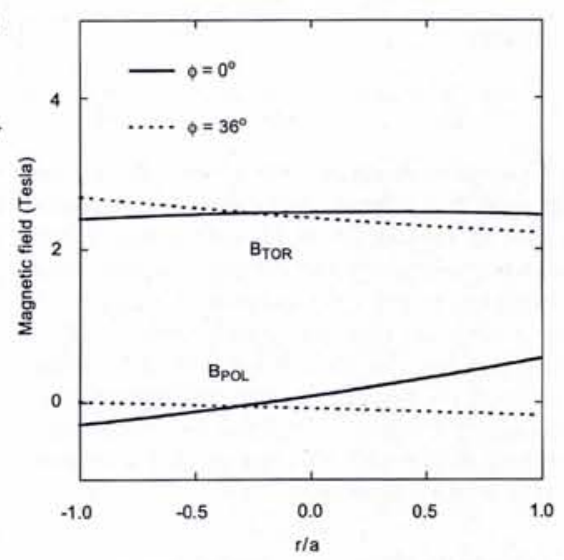

Fig 1d Spatial variation of the toroidal and poloidal fields in a stellarator. Values at two toroidal positions are shown $\left(\phi=0^{\circ}\right.$ and $\left.\phi=36^{\circ}\right)$ to illustrate the toroidally asymmetric nature of a stellarator plasma-the tokamak and RFP profiles are the same for any toroidal position tokamak following the announcement in 1968 , by a group at the Kurchatov Institute in Moscow, that plasmas in the T-3 tokamak had reached the then unheard of temperature of $1 \mathrm{keV}$. Since 1968 tokamak dimensions have increased from major and minor radii of 1 metre and 0.12 meters in T- 3 to the region of 3 and 1 meters respectively in the current generation of large tokamaks, represented by JET in the UK and the Japanese JT-6oU experiment in Naka. The plasma volume has increased from $\sim 0.3 \mathrm{~m}^{3}$ to $\sim 100 \mathrm{~m}^{3}$ and the current carrying capability, which has an important bearing on plasma confinement in tokamaks, from $60 \mathrm{kA}$ to $7 \mathrm{MA}$. The parallel development of high power plasma heating systems has allowed the central plasma electron temperature to be increased from $1 \mathrm{keV}$ in $\mathrm{T}-3$ to above $10 \mathrm{keV}$ in JET, JT-6oU, and the US TFTR device in Princeton. The increase in central ion temperatures into the range 30$40 \mathrm{keV}$ has been even more spectacular.

Until the first plasmas were produced early in 1998 in the new Japanese LHD stellarator in Toki the largest stellarators had a plasma volume of $\sim 1 \mathrm{~m}^{3}$, comparable to that of the generation of tokamaks constructed in the late 1970s. The advent of LHD, and the Wendelstein 7-X ( $\mathrm{W}_{7}-\mathrm{X}$ ) experiment, which is under construction at Greifswald, Germany (see Europhysics News 263 1995), both with plasma volumes of $\sim 30 \mathrm{~m}^{3}$, will bring stellarators into line with the largest of the current generation of medium-sized tokamaks, such as ASDEX Upgrade in Garching, Germany, Tore Supra in Cadarache, France, and DIII-D in San Diego. It is not surprising, therefore, that the highest ion and electron temperatures obtained in stellarators are $1.5 \mathrm{keV}$ and $5.8 \mathrm{keV}$ respectively, values which were achieved in Wendelstein 7-AS (W7-AS) in Garching.

Although RFPs such as MST in Madison and RFX in Padua, Italy, have plasma volumes of 6-8 $\mathrm{m}^{3}$ and plasma current capabilities of 1-2 MA, and are therefore equivalent in these respects to a medium-sized tokamak, the maximum ion temperature achieved to date has been $\sim 1 \mathrm{keV}$. This reflects the poorer confinement quality of RFP plasmas, which is due in part to the lower magnetic field and in part to the magnetic relaxation (dynamo) processes associated with the formation of the reversed field equilibrium.

\section{Plasma heating and confinement}

To attain the necessary plasma parameters for ignition, where $\alpha$-particle heating is sufficient to maintain the plasma temperature, the plasma must be heated in some way. In tokamaks and RFPs, ohmic heating 
is unlikely to be adequate to reach ignition, while stellarators lack even this elementary form of heating. Four auxiliary plasma-heating techniques have therefore been developed to an advanced level-the diversity of approaches illustrates the links that fusion research has established with many other areas of physics.

Intense beams of neutral particles with energies in the $100 \mathrm{keV}$ range and total powers of up to $40 \mathrm{MW}$ can be injected across the magnetic field so that they collide with the plasma particles, are ionized, confined, and slow down, transferring their energy to the background plasma.

Plasmas can also be heated by RF waves using the variety of resonant absorption processes which occur. Ion cyclotron heating operates at frequencies in the range 30-120 MHz; lower hybrid wave heating, named after a coupled resonance of the ions and electrons, operates in the region of $1-8 \mathrm{GHz}$; and electron cyclotron resonance absorption involves frequencies in the region of $30-140 \mathrm{GHz}$. RF systems capable of launching up to $20 \mathrm{MW}$ for many seconds are operational. The most significant application of lower hybrid wave heating in modern tokamak experiments is in the driving of plasma current, since a toroidally asymmetric wave spectrum can be launched, which imparts a net momentum to the electron population. The other three heating schemes also have a current drive capability, but their efficiency (the ratio of driven current to input power) is lower than that of lower hybrid waves.

For ignition it is necessary not only to achieve high temperatures, but to retain energy within the plasma for sufficiently long that the rate of $\alpha$-particle heating balances the power lost by conduction, convection and radiation. In toroidal confinement systems magnetic field lines close-up within the torus and power is then lost by transport across field lines. The irreducible minimum transport rate is then set by Coulomb collisions between plasma particles. Toroidal geometry can play a significant role in these processes since a fraction of the particles can be trapped by magnetic mirrors within the plasma. Indeed, a particle following a helical field line sees a magnetic field of varying intensity and will be reflected when reaching a region of stronger magnetic field if it has insufficient velocity parallel to the field line. These 'trapped particles' are well confined in a tokamak, but their existence can be inferred from macroscopic measurements. In stellarators the toroidal periodicity of the plasma gives rise to helically trapped particles, whose orbits deviate significantly from surfaces formed by the magnetic field lines and can even intersect the wall of the plasma chamber. The configuration optimization approach exploited in 'advanced stellarators', which will be implemented in the $\mathrm{W}_{7}$ - $\mathrm{X}$ experiment is expected to minimize the loss of such particles.

The actual transport losses measured in tokamaks and stellarators turn out to be similar to each other, but much worse than originally expected. In particular, in tokamaks the rate of heat loss through the electrons is approximately two orders of magnitude greater than predicted on the basis of 'neoclassical theory', which derives heat and particle transport rates from the underlying Coulomb collisions, taking the effects of toroidal geometry touched on in the previous paragraph into account. It is believed that the enhanced rates of transport losses are due to smallscale instabilities with wavelengths very

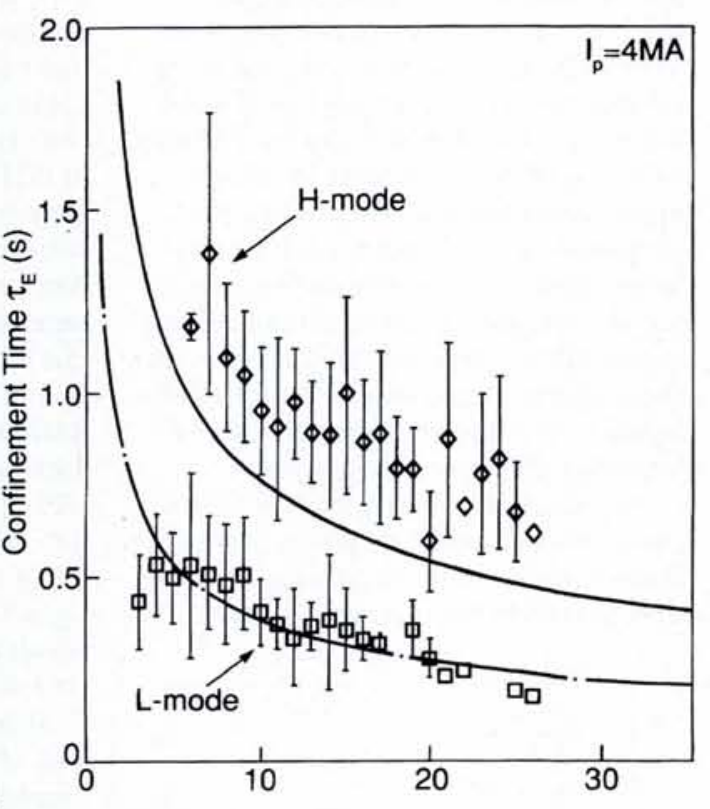

Fig 2 Energy-confinement time plotted against the input power for a series of $\mathrm{L}$ - and $\mathrm{H}$-mode plasmas in the JET tokamak. The lower curve (dashed) is derived from an empirical energy-confinement time scaling in which the confinement time varies inversely with square root of the input power, while the upper line corresponds to twice this scaling. This illustrates that $\mathrm{H}$-mode energy confinement is approximately a factor of 2 higher than Lmode energy confinement, but that the scaling with plasma parameters is broadly similar for the two regimes (courtesy JET Joint Undertaking)

much smaller than the plasma minor radius. The proposed instabilities are driven by the free energy available in the plasma pressure and magnetic field profiles (see Romanelli and Hidalgo, chapter 3.1 and Garbet chapter 3.5).
In the absence of a quantitative understanding of the processes determining transport, a more empirical approach to the characterization of plasma confinement has been developed. This relates the global-energy-confinement time of a plasma configuration to macroscopic plasma parameters, and has the merit that it offers a route to determining how large a device is required to be in order to achieve the energy-confinement time required for ignition. Confinement in the majority of tokamak plasmas can be described by an energy-confinement time scaling which depends linearly on the plasma current, the 1.5 power of the characteristic length scale (eg the major radius), and inversely on the total input power raised to a power between $1 / 2$ and $2 / 3$. There are also other, less significant, dependences. Intriguingly, this type of confinement behaviour, known as low mode, or L-mode, finds a parallel in the confinement behaviour in stellarators when the plasma current is replaced by the magnetic field. In RFPs the high level of magnetic fluctuations in the plasma interior associated with the sustainment of the configuration means that the plasma thermal insulation is provided by a thin layer at the plasma edge. As a result, energy-confinement times in the largest RFPs are only a few milliseconds, or 1-2 orders of magnitude smaller than that in tokamaks of equivalent size and current.

While L-mode confinement represents the 'lowest common denominator' of tokamak and stellarator confinement, many plasma regimes have been identified in which the global energy confinement exceeds the predictions of L-mode scaling by a factor of 2 or more. The most reproducible of these is the high confinement regime, or $\mathrm{H}$ mode, which was first observed in 1982 in the ASDEX divertor tokamak in Garching. Energy confinement is typically twice as high as L-mode, though the scaling with plasma parameters is broadly similar in the two regimes (figure 2). In recent years this regime has also been observed in the W7-AS stellarator and, although the improvement in energy confinement is not as great as in tokamaks, the details of the transition and subsequent plasma behaviour appear very similar in the two systems.

The physics processes which produce the $\mathrm{H}$-mode transition are not yet fully understood (see Romanelli and Hidalgo, chapter 3.1), but it is clear that the transition originates at the plasma edge. Here the temperature and density profiles become very steep in a narrow layer, perhaps only several centimetres wide, and 
strong poloidal and toroidal plasma flows can occur. A widely accepted explanation of the H-mode transition is that in the narrow edge region the development of a gradient in the radial electric field, whose origin is not understood, produces sheared plasma flows as a result of the $\mathrm{E} \times \mathrm{B}$ drift. These flows can suppress the growth of electrostatic instabilities thought to be responsible for the anomalous transport in L-mode plasmas (see Romanelli and Hidalgo, chapter 3.1). Recent measurements in RFX have shown that a strongly sheared plasma flow can occur in the edge region of an RFP plasma, suggesting that turbulent transport can be quenched by a similar mechanism to that observed in tokamaks and stellarators.

In fusion power plants $3.5 \mathrm{MeV} \alpha$-particles produced by the DT reactions give rise to self-heating of the plasma-an effect observed for the first time in magnetically confined plasmas in recent DT experiments in TFTR and JET (see Keilhacker and Watkins, chapter 4.4). Alpha particles must therefore be well confined to ensure efficient plasma heating and to protect the first wall of the confinement chamber from bombardment by high energy particles. The identification and analysis of possible sources of anomalous losses of $\alpha$-particles have been particularly fruitful areas of interaction between experiment and theory. Experimental evidence has come from the many devices in which high energy ion populations produced by additional heating systems have been used to simulate $\alpha$ particle populations, as well as from the TFTR and JET DT experiments. This has provided extensive benchmarking of theoretical predictions and, although some areas of theoretical uncertainty remain, extrapolation to experiments such as ITER (see Parker, chapter 4.3) indicates that $\alpha$ particle confinement will be acceptable in ignited plasmas.

\section{Magnetohydrodynamic stability} Magnetohydrodynamic (MHD) theory describes the stability properties of a plasma fluid immersed in a magnetic field. Two general classes of instabilities can be identified. Ideal instabilities would occur even if the plasma were perfectly conducting and they involve motions in which the plasma and magnetic field are essentially 'frozen' together. Resistive instabilities involve the small but finite plasma resistivity - typical plasmas in modern fusion experiments have a resistivity of the order of that of room temperature copper $\left(\sim 2 \times 10^{-8} \Omega \mathrm{m}\right)$. These instabilities can involve rearrangement of the magnetic field topology by tearing and reconnec- tion of the field lines. The theory of MHD stability of toroidal plasmas is highly developed and provides an excellent qualitative, and in many cases quantitative, description of plasma behaviour.

For fusion power plants the attainable plasma beta, $\beta$, is a central issue (see Villard, chapter 3.2). This is a normalized measure of the plasma pressure, defined as the ratio of the average plasma pressure to the magnetic field pressure. Beta values of several per cent or greater, corresponding to a plasma pressure of $\sim 3 \times 10^{5}$ Pascal (or several times atmospheric pressure), must be achieved if magnetic fusion power plants are to be economically viable. In the DIII-D tokamak in San Diego, values of $\beta$ as high as $12.5 \%$ have been achieved for periods much shorter than 1 second and values of $8 \%$ for periods of $\sim 1$ second, while the 'spherical tokamak' START at the Culham Laboratory (see Morris, chapter 5.2) has reached $\beta$ values of up to $40 \%$ for about $1 \mathrm{~ms}$. Beta can be limited by either ideal or resistive modes, depending on the current and pressure profiles.

In RFPs, $\beta \sim 10 \%$ is typical because the relaxation process sustaining the field reversal acts to maintain stability against $\beta$-limiting MHD modes. This attractive feature of RFPs has sustained the longterm interest in the development of this configuration. In stellarators the $\beta$-limit is set by constraints on the global equilibrium rather than by MHD instabilities. The force balance between plasma pressure and magnetic field in a toroidal system produces currents parallel and perpendicular to the magnetic fields lines, though no net toroidal current results. The parallel current component causes a change in the plasma equilibrium, essentially a shift of the plasma column along the major radius which increases with $\beta$. As a result, the maximum $\beta$ achieved in stellarators is $\sim 2.2 \%$, obtained in CHS at Nagoya. The $\beta$ induced equilibrium shift can be minimized by a process which is closely related to the optimization technique discussed previously. Thus $\beta$ values as high as $5 \%$ are considered possible in the $\mathrm{W}_{7}-\mathrm{X}$ stellarator. The theoretical limit in the TJ-II stellarator at Madrid is even higher (see Alejaldre, chapter 5.1).

Exceeding pressure, density, or current limits in tokamak plasmas can give rise to various MHD instabilities of which the most serious is the major disruption. This event, which can be triggered by resistive or ideal instabilities, typically quenches the plasma thermal energy in less than $1 \mathrm{~ms}$ and the plasma poloidal magnetic energy in less than $10 \mathrm{~ms}$. Although these timescales may be up to an order of magnitude longer in tokamak power plants, the total energy involved in each stage will be $\sim 1$ GJ, so that some areas of the first wall of the chamber will be exposed to high energy loads $\sim 100 \mathrm{MJm}^{-2}$. In addition, inductive effects could give rise to high current pulses, of order $10 \mathrm{MA}$, in the metallic structure of the plasma chamber. Large electromechanical forces, $\sim 10^{4}$ tonnes, would result. Experiments are underway to develop techniques which can dissipate the thermal and magnetic energy of the plasma in a short timescale to avoid the most severe disruptive effects. It is regarded as an important advantage of stellarators and RFPs that neither suffers from this form of instability.

\section{Power and particle exhaust}

In a fusion power plant the several hundred MW of power deposited by $\alpha$-particles must be exhausted in a way which does not exceed the power handling capa-

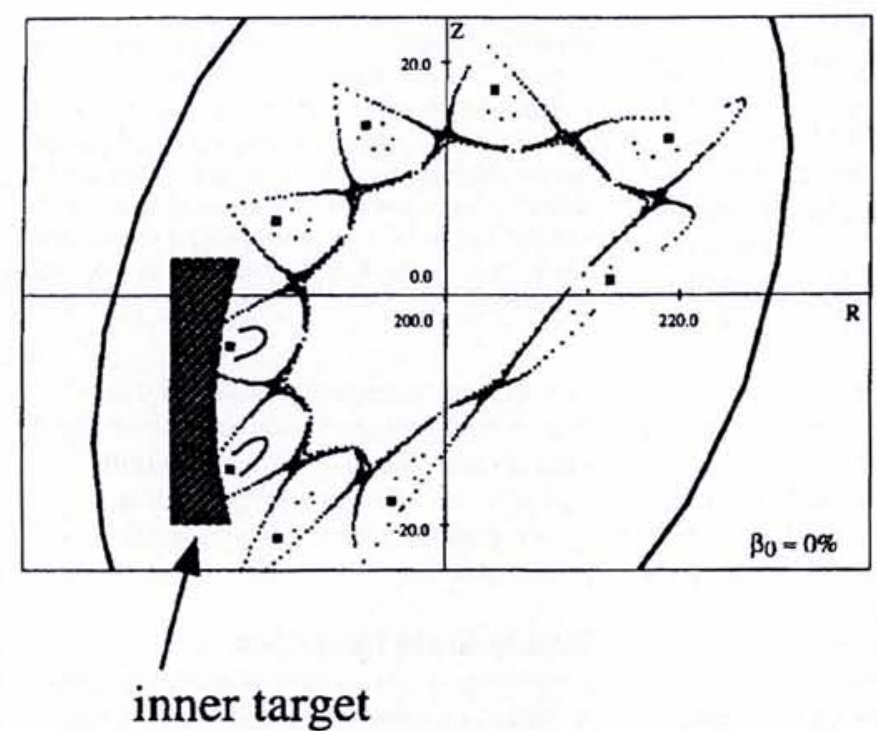

Fig 3 Poloidal cross-section of the 'island divertor' geometry in the Wendelstein 7-AS stellarator in Garching in Germany, showing the magnetic configuration with a ring of magnetic islands at the plasma edge which act as localized divertors when they interact with a material target 
bility of the first wall, $\sim 5-10 \mathrm{MWm}^{-2}$, and which does not lead to excessive impurity production which would contaminate the plasma and quench the thermonuclear burn. Impurity production also causes erosion of first wall materials and this must be limited to ensure an adequate lifetime for the surfaces in contact with the plasma edge. The thermalized $\alpha$-particles, or 'helium ash', must in addition be pumped away from the plasma edge to avoid their poisoning the plasma and slowing the reaction rate.

In present experiments, where there is little thermonuclear power, two solutions to the power handling problem have been developed. In the 'limiter' concept a specially prepared surface intersects the helical field lines just inside the bore of the toroidal vacuum chamber and defines the plasma edge. In the 'divertor' concept the plasma boundary is defined by a magnetic surface known as a 'separatrix', which is formed by creating a position of zero field (a 'null' or 'X-point') in the poloidal field distribution by means of external coils. Field lines outside the separatrix then intersect a 'target plate' (see Schneider, chapter 3.3).

Power handling limitations are similar for divertors and limiters and an additional factor is needed. The solution, devised initially in the TEXTOR tokamak in Jülich in Germany, is to radiate as much of the plasma power as possible, virtually 100 per cent in some experiments, by 'seeding' the plasma with small quantities of noble gases such as neon. Such impurities enhance the radiation primarily from the plasma edge, which limits their contribution to transport in the plasma core. In this way the power is effectively redistributed over the entire wall of the vacuum chamber, reducing the power handling problem to a manageable level. Nevertheless, major challenges remain in reducing the impact of the radiation on plasma confinement and in minimizing the contamination of the plasma by the radiating impurities.

Stellarator and RFP research has focussed more on the development of core plasma performance and is only now addressing the problem of power exhaust. In one class of stellarator, the heliotron, a 'helical divertor' structure can be formed naturally in the magnetic field at the plasma edge. This configuration has been investigated in the Heliotron-E experiment in Nagoya and is being exploited in
LHD. An alternative concept, the 'island divertor', which is formed when an appropriate value of the edge rotational transform is selected, has been demonstrated successfully on the W7-AS stellarator (figure 3 ) and is planned for $\mathrm{W}_{7}-\mathrm{X}$. Control of the plasma-wall interaction in RFPs is less well developed, though it is now receiving increased attention. While the majority of RFPs operate with a limiter, a divertor configuration has been studied in TPE-2M in Tsukuba in Japan.

Exhaust of helium ash from the plasma must be rapid enough so that the residence time of helium in the vacuum confinement time. To achieve this helium ions must diffuse sufficiently rapidly across the plasma and then the neutralized helium must be pumped away. It has been demonstrated on several tokamaks that helium injected into the plasma by neutral beams or by gas puffing is transported across the plasma at the same rate chamber is at most 5-10 times the energy-

advantage in the context of steady-state operation since there is no requirement for an externally driven current in the plasma. The superconducting coils of the LHD and W-7X experiments will allow a steady-state magnetic configuration to be established for the first time, so that the remaining physics issues required to establish the credibility of these systems as power plants can be addressed-adequate confinement scaling to keep the size of ignition experiments within practical limits, acceptable divertor operation to provide the requisite power handling and particle control, and the demonstration of the required plasma $\beta$ for economic power production.

Both RFPs and tokamaks use a toroidal plasma current to establish the plasma equilibrium and a source of current other than that produced by transformer action is required for steady-state operation. In RFPs, moreover, the magnetic turbulence associated with the dynamo responsible for field reversal is thought to contribute significantly to the poor energy confinement observed. Reducing the level of this turbulence is therefore the major challenge facing research on RFPs. External poloidal current drive by pulsing of the toroidal field coils, called pulsed poloidal current drive (PPCD), has been studied on the MST and RFX devices. These experiments have shown that the magnetic fluctuations measured at the plasma edge are strongly reduced, MHD activity is suppressed, and energy confinement can improve by up to a factor of 5 . Figure 4 illustrates the associated improvement in central electron temperature observed in RFX due to the reduction in core magnetic fluctuations.

Providing a steady-state current drive Fig 4 Radial profiles of electron temperature measured in the RFX reversed field pinch at the Istituto Gas lonizzati del CNR in Padua. Temperature profiles in normal operation (squares) and during pulsed poloidal current drive operation (triangles) are compared. This illustrates the improvement in core plasma confinement which is obtained during pulsed poloidal current drive operation as a result of the reduction in magnetic fluctuations

as other particles and that sufficiently high neutral pressure can be obtained at the plasma edge to sustain the helium pumping speed ( $1.5 \mathrm{~Pa} \mathrm{~m}^{3}$ per GW of fusion power) required for tokamak power plants.

\section{Steady-State Operation}

Among the three principal magnetic confinement systems stellarators have a clear capability is one of the most significant issues facing tokamak research and this has been addressed by exploiting the current drive capability of additional heating plasma currents of up to 3.6 MA for severusing about $10 \mathrm{MW}$ of lower hybrid waves. This technique has also been used to produce a 2 hour pulse at $20 \mathrm{kA}$ in the TRIAM-1 tokamak in Kyushu and a 120 second pulse at $800 \mathrm{kA}$ in Tore Supra. Nevertheless, at the current drive efficiencies expected in tokamak power plants, several $100 \mathrm{MW}$ of additional heating power would be required to achieve full non-inductive current drive, which is unrealistic from an economic point of view. systems. For example, externally driven al seconds have been obtained in JT- $60 \mathrm{U}$ 


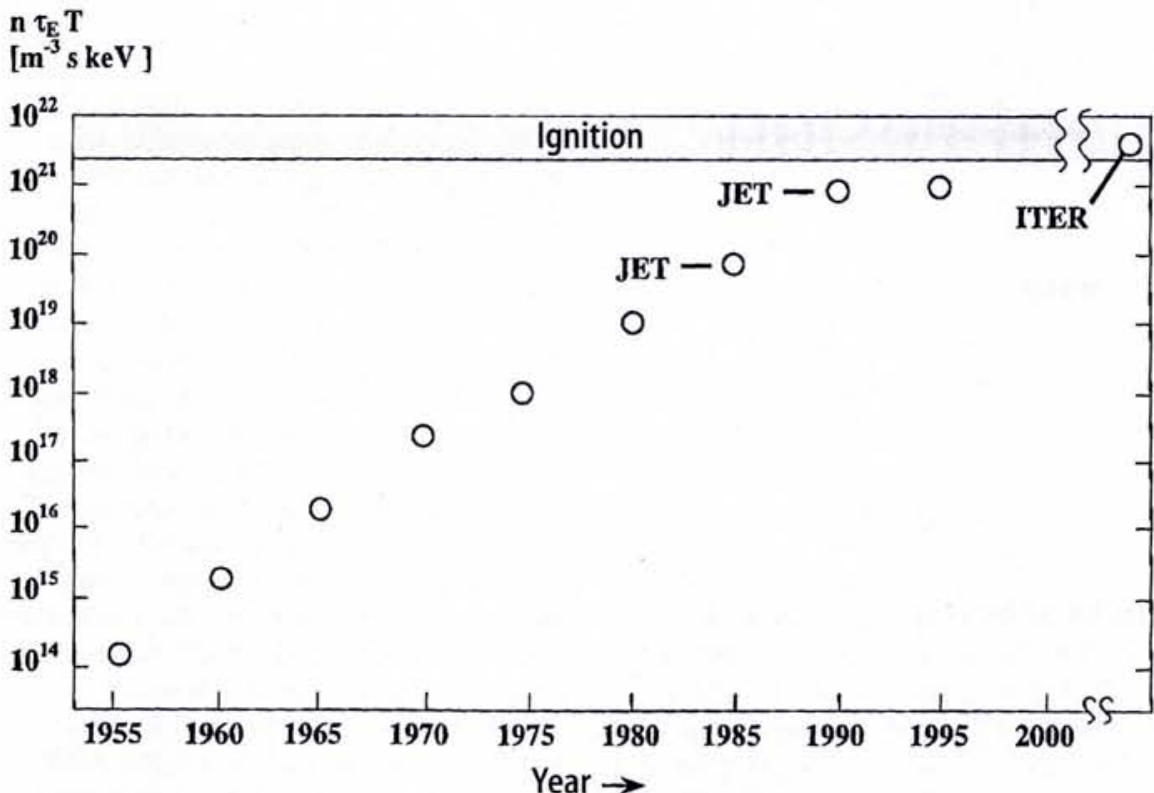

In tokamaks, therefore, the challenge of providing a steady-state current drive capability would be daunting were it not for an important plasma-induced effect: the 'bootstrap current'. This is a toroidal current, driven by the radial pressure gradient, which arises from the exchange of momentum between trapped and 'passing' (ie non-trapped) particles in a toroidal confinement system. Its existence is a further experimental confirmation of the neoclassical theory of plasma behaviour discussed earlier (see plasma heating and confinement in this chapter). In specially prepared discharges in the largest tokamak experiments up to 80 per cent of the plasma current has been driven in this way. Steady-state current drive could thus be achieved in a tokamak power plant by driving the remaining fraction of the current using one or more of the additional heating systems at a power level of $\sim 100 \mathrm{MW}$, which would be both practical and economically viable.

Significant progress has been made recently in developing a tokamak plasma regime which combines important elements required for steady-state operation. Plasmas have been established in which the $q$-profile has a minimum at approximately $1 / 3$ to $1 / 2$ of the minor radius and then remains flat, or increases, towards the plasma centre. The transport losses at the plasma centre then reduce substantially and the MHD stability improves. There is some evidence that the reduced transport is associated with the development of an increased electric field gradient near the plasma centre which gives rise to an internal transport barrier'. The high confinement then leads to the development of a high pressure gradient, on which the bootstrap current depends. In present experiments this plasma regime is usually transient, lasting only 1 second or so, and its properties have not yet been fully characterized. Nevertheless, its existence points the way to the development of a plasma regime (the 'advanced tokamak scenario') with better confinement than is currently achieved together with good MHD stability and a high fraction of bootstrap current.

\section{Status}

The progress in magnetic fusion research is shown in figure 5, which shows the steady increase in the fusion performance parameter, $n \tau_{E} T$ (the product of plasma density, energy-confinement time, and ion temperature) during the last 40 years. Thi must reach a value of $\sim 5 \times 10^{21} \mathrm{~m}^{-3} \mathrm{skeV}$ for a plasma to achieve ignition. The increasing scale of magnetic confinement experiments, together with the accompanying improvements in the understanding of the physics of magnetoplasmas, has raised the values attained experimentally by 7 orders of magnitude and has brought the field to the present point, where the largest experiments are within a factor of 5 of the required value. On this basis it can be expected that the parameters of the ITER tokamak (see Parker, chapter 4.3) are adequate to ensure that ignition will be achieved.

Three principal conclusions can be drawn about the present status of magnetic confinement fusion. Firstly, there is now substantial, though still incomplete, understanding of plasma behaviour in the principal toroidal confinement configurations, and there is a much deeper appreciation of the complexity of the physics of high temperature magnetoplasmas. Secondly, new opportunities for further improvement in plasma performance are opening with the advent of a new generation of large stellarators such as LHD, the development of 'advanced tokamak scenarios', which may offer a viable route to
Fig 5 Progress in the fusion performance parameter, $n \tau_{\varepsilon} T$, during the last 40 years of magnetic confinement research $-n$ is the central plasma density, $\tau_{E}$ the energy-confinement time, and $T$ the central ion temperature. This parameter must reach a value of $\sim 5 \times 10^{21} \mathrm{~m}^{-3} \mathrm{skeV}$ for a plasma to achieve ignition. The largest experiments, JET, JT-60U and TFTR have achieved values of $\sim 1 \times 10^{21} \mathrm{~m}^{-3} \mathrm{skeV}$. The apparent saturation of the trend in recent years reflects the fact that these large experiments are essentially at the limit of their capability and that a new device, such as ITER (for which the predicted value is shown), is required to produce the parameters necessary for an ignited plasma (courtesy J.A. Wesson Tokamaks second edition, Oxford University Press, 1997)

steady-state tokamak operation, and the realization of a variety of new tools for enhancing plasma performance in RFPs. Finally, given the production of over $10 \mathrm{MW}$ of DT fusion power in TFTR and $16 \mathrm{MW}$ in JET, plasma performance in tokamaks has advanced to the point where the construction of a DT-burning plasma experiment such as ITER would be a timely next step.

The key issues and achievements in magnetic confinement research have been reviewed briefly here, and it has been possible to touch on only a very few of the impressive achievements in experimental, theoretical, and computational physics, as well as in engineering, which have accompanied the development of toroidal confinement systems to their present level of sophistication. In particular it has not been possible to illustrate adequately that magnetic confinement fusion research has proved an extremely fertile meeting ground for many branches of physics and engineering.

The author would like to thank Rosario Bartiromo of the Istituto Gas Ionizzati del CNR in Padua, Fritz Wagner of the MaxPlanck-Institut für Plasmaphysik in Garching, R. Koch of École Royale Militaire in Brussels, and also L. Giannone, A. Loarte, and E. Righi for preparing several of the figures for this review.

\section{Further reading}

R.J. Goldston, P.H. Rutherford Introduction to Plasma Physics (Institute of Physics Publishing, Bristol, 1995)

R.O. Dendy editor Plasma Physics: An Introductory Course (Cambridge University Press, 1993)

J.A. Wesson Tokamaks second edition (Oxford University Press, 1997)

J.Sheffield The Physics of Magnetic Fusion Reactors Rev. Mod.Phys. 66 (1994) 1015 